\title{
PERFORMANCE OF SOME FLAX VARIETIES (Linum usitatissimum L.) UNDER DIFFERENT SOWING AND HARVISTING DATES: 2- SEED YIELD AND ITS COMPONENTS
}

\author{
Kandil, A. A.*; A. A. Lellah*; T. A. Abou-Zaied ${ }^{\star *}$ and \\ Heba A. H. Turky ** \\ * Agronomy Dept., Fac. of Agric., Mansoura University \\ ** Fiber Crop Res., A.R.C
}

\begin{abstract}
Two field experiments were conducted at El-Gemmeiza Res. Station. Gharbiua Governorate Agric. Res. Center during the two successive seasons of 2006/2007 and $2007 / 2008$. The objectives of this research was aimed to study the performance of some flax varieties i.e. Sakha 2, Sakha 3, Sakha 4 and Giza 8 under different sowing dates (on $20^{\text {th }}$ October, $10^{\text {th }}$ November, $1^{\text {st }}$ December) and harvesting dates (after 135, 150 and 165 days) from sowing and their effects on seed yield and its components. The main findings could be summarized as follows.

Early sowing on $20^{\text {th }}$ October significantly increased seed yield/ plant in the first season. Sowing flax on $10^{\text {th }}$ November significantly increased seed yield/ faddan as compared with the other sowing dates.

Delaying harvesting date to 150 days from sowing significantly increased number of capsules/ plant in the second season and delaying harvesting date to 165 days from sowing significantly increased number of capsules per plant in the first season, 1000 seed weight, seed yield per plant and seed yield per faddan in the both seasons.

Sakha 3 and Sakha 4 varieties surpassed the other varieties in number of seeds per capsules in both seasons. Sakha 2 and Giza 8 varieties surpassed the other varieties in the seed yield characters, except number of seeds per cpsules . Giza 8 superior the other varieties bor number of capsules/ plant in the first season, 1000 seed weight, seed yield/ plant and seed yield/ faddan in both seasons.

It could be concluded that sown Sakha 2 or Giza 8 early on $10^{\text {th }}$ November and harvesting it after 165 days from sowing maximized seed yield/ fad. under the environmental conditions of Garbiua Governorate.
\end{abstract}

\section{INTRODUCTION}

Flax (linum usitatissimum L.) is still a major baste fiber crops in Egypt. It is known as long as pharaoh civilization and it is grown in large scale around the world. In northern region, it is grown as a fiber crop, in southern ones and cultivated as Mediterranean sea region. It is grown as a dual purpose crop for either fibers extracting from stems by retting after soaking in water.

In Egypt during the last few years, by releasing new varieties characterized gave high yield ability and best quality in addition to improve different agricultural practices for this crop. With respect varietal differences, Abdel-Fatah (1994) and Kineber (1994) showed that seed yields as well as its components differed significantly among flax varieties. El- Kady (1995) reported that varieties differed significantly in all characters of flax.

According to sowing date, Dixit et al.(1994) reported that delaying sowing date after $25^{\text {th }}$ October decreased seed yield. They also found that, 
Kandil, A. A. et al.

seed yield was decreased by $75 \%$ when flax was sown on $15^{\text {th }}$ December. Moawed (1996) reported that when flax sown on the first 15 days from November gave the highest number of capsules per plant, number of seeds per capsule, seed yield per plant or faddan. Salama (1996) reported that early planting date on $15^{\text {th }}$ November significant increased number of capsules, 1000-seed weight, seed yield as well as oil percentage than the late planting on first December. Mohamed et al. (1998) observed that yield attributes viz number of capsules/ plant, number of seeds/ capsules, seed yield/ plant and 1000 seed weight were higher in the first early date on 21 October. Also, they observed that the oil percentage was higher at the first and second sowing dates, ( $20^{\text {th }}$ October and $10^{\text {th }}$ November) thereafter it declined with delaying sowing date. El-Deeb and Abd-El Fataha (2006) reported that sowing date had a significant effect on all characters, except 1000 -seed weight and oil percentage. Sowing flax on $15^{\text {th }}$ November recorded the highest mean of seed yields as well as its components. Abd-ElDaiem (2009) found that sowing flax on $25^{\text {th }}$ October gave higher values seed yield/ plant, seed yield faddan, as well as per faddan, number of capsules/ plant, number of seeds/ capsule and 1000 seed weight. There ware also a gradual increase in oil percentage with delaying in sowing date until $9^{\text {th }}$ November.

Harvesting date play a great role in fiber length and quality of flax as well as seed yield. In this respect, Mostafa (1990) reported that there was a continuous increase in seed yields as well as its components with delaying harvesting date from 130 to 170 days from sowing. Abd-El-Daiyme et al. (2007) found that delaying harvesting date up to the oldest age (170 days from sowing) caused increasing in all seed yield characters in addition to all mineral contents of flax seeds.

The main objectives of this work is aimed to determine the effect of sowing and harvesting dates on growth, yield and quality of four flax varieties i.e., Sakha 2, Sakha 3, Sakha 4 and Giza 8 under the environmental conditions of Gahrbuia gouernorat.

\section{MATRIALS AND METHODS}

The present investigation was carried out at El- Gemmeiza agricultural Research Station, Agric .Res. Center, Gharbiua Governorate, Egypt, during the two successive seasons of 2006/ 2007 and 2007/2008. The objectives of this research was aimed to study the effect of sowing and harvesting dates on yield and its components of some flax (Linum usitatissimum L.) varieties i.e. Sakha 2, Sakha 3, Sakha 4 and Giza 8.

Three tested sowing dates planting on $20^{\text {th }}$ October, $10^{\text {th }}$ November and $1^{\text {st }}$ December were conducted in three separate experiments. A split plot design with three replications for each sowing date was designed. The main plots were devoted to the three harvesting dates at 135,150 and 165 days after sowing as shown in Table 1. The sub plots were assigned to the following four varieties Sakha 2, Sakha 3, Sakha 4 and Giza 8 are shown in Table 2. 
Table 1: Time of harvesting dates at the three studied sowing dates.

\begin{tabular}{|l|c|c|c|}
\hline \multirow{2}{*}{ Sowing date } & \multicolumn{3}{|c|}{ Harvesting dates } \\
\cline { 2 - 4 } & after 135 days & after 150 days & after 165 days \\
\hline $20^{\text {th }}$ October & March 5 & March 25 & April 5 \\
\hline $10^{\text {th }}$ November & March 25 & April 10 & April 25 \\
\hline $1^{\text {st }}$ December & April 15 & May 1 & May 15 \\
\hline
\end{tabular}

Table 2: pedigree of studied varieties.

\begin{tabular}{|l|c|}
\hline Varieties & Source \\
\hline Sakha 2 & Hera $\times 1.123$ \\
\hline Sakha 3 & (Belinka $(2 E) \times 1.2096)$ \\
\hline Sakha 4 & (Belinka $(\mathrm{R3}) \times 1.2569$ \\
\hline Giza 8 & (Giza6 $\times$ Senta Catalina \\
\hline
\end{tabular}

At each harvesting date, ten guarded plants were taken at random from each sub- plot to be used in recording the straw yield component characters of flax. Flax for seed yield per faddan was recorded on the whole sub plot area basis. The following seed yield and its components were determined.

2- Number of seeds/ capsule 1- Number of capsules/ plant

3-Seed yield/ plant

4- Seed yield/ faddan $(\mathrm{Kg})$

5-1000 seed weight

The obtained data for each planting date was statistically analysis separately by technique of analysis of variance thereafter, combined analysis for collected data of all sowing dates in both seasons were subjected to the statistical analysis according to the procedures outlined by Gomaz and Gomaz (1984). The treatment means were compared using the Newly Least Significant Difference (NLSD) test which developed by Waller and Dunckan (1969). Field experiment was carried out each season using split-plot design, where each sub- plot size was $6 \mathrm{~m} 2(2 \times 3 \mathrm{~m})$. was corn (Zea Maize) crop previous in both seasons. The experimental field area was well prepared. Calcium super phosphate at a rate of $100 \mathrm{Kg} /$ faddan $\left(15.5 \% \mathrm{p}_{205}\right)$ before sowing and potassium sulphate $(48 \% \mathrm{~K} 20)$ was applied at the rat $50 \mathrm{Kg} /$ faddan in one dose. Nitrogen was added at the rate $45 \mathrm{~kg} \mathrm{~N} /$ Faddan in the form of urea $(46 \% \mathrm{~N})$ in two dose, the first half was added be fore the first irrigation

\section{RESULTS AND DISCUSSION}

\section{Sowing date effects.}

Results of seed yield and elated characters for the three sowing dates showed significant affected as shown in Table 3 . The results showed that Seed yield / plant and Seed yield/ faddan significantly affected by sowing dates. Sown on $20^{\text {th }}$ October gave the heighest seed yield/ plant in the first season while sown on $1^{\text {st }}$ December gave the heighest seed yield/ plant in the second season. Sown on $10^{\text {th }}$ November came in the first ranke for seed yield per faddan during two seasons. However, the lowest seed yield / plant and seed yield/ faddan was obtained on $20^{\text {th }}$ October in the second season. The highest values in seed yield characters were obtained with the early sowing date may be attributed to the suitable climate conditions 
Kandil, A. A. et al.

i.e. the more favorable temperature, day length and the longer duration of the growth period. These results are in harmony with those obtained by Salama (1996), Mohamed et al. (1998) and Abo Zaied and Mousa (2007).

\section{Harvesting date effects.}

With respect to the effect of seed yield and its components, results in Table 3 indicated that there were significant differences among the three harvesting dates on number of capsules per plant, seed index, seed yield per plant and seed yield per faddan in the two seasons. The harvesting date after i.e. 165 days from sowing gave the highest number of capsules per plant in the first season, seed index, seed yield per plant and seed yield per faddan in both seasons. The highest values of number of capsules per plant were recorded from harvesting date after 150 days from sowing in the second season. These results are mainly due to the fact that plants did not reach their physiological maturity till 165 days from sowing and plants continued to increase in height consistently from 135 to 165 days old.

Table 3: Means of number of capsules/plant and number of seeds/ capsule 1000 seed weight, seed yield / plant and seed yield/ faddan $(\mathrm{kg})$ as affected by sowing and harvested dates and flax varieties during 2006/2007and 2007/2008 seasons.

\begin{tabular}{|c|c|c|c|c|c|c|c|c|c|c|}
\hline \multirow{2}{*}{$\begin{array}{l}\text { Characters } \\
\text { Treatment/ } \\
\text { seasons }\end{array}$} & \multicolumn{2}{|c|}{$\begin{array}{c}\text { Number of } \\
\text { capsules/ plant }\end{array}$} & \multicolumn{2}{|c|}{$\begin{array}{c}\text { Number of } \\
\text { seeds/capsule }\end{array}$} & \multicolumn{2}{|c|}{$\begin{array}{c}1000 \text { seed } \\
\text { weight }\end{array}$} & \multicolumn{2}{|c|}{$\begin{array}{c}\text { Seed yield / } \\
\text { plant }\end{array}$} & \multicolumn{2}{|c|}{$\begin{array}{l}\text { Seed yield/ } \\
\text { faddan }(\mathrm{kg})\end{array}$} \\
\hline & $\begin{array}{l}2006 / \\
2007\end{array}$ & $\begin{array}{l}2007 / \\
2008\end{array}$ & $\begin{array}{l}2006 / \\
2007\end{array}$ & $\begin{array}{l}2007 / \\
2008\end{array}$ & \begin{tabular}{|l|}
$2006 / 1$ \\
2007
\end{tabular} & $\begin{array}{l}2007 / \\
2008\end{array}$ & $\begin{array}{l}2006 / 1 \\
2007\end{array}$ & $\begin{array}{l}2007 / \\
2008\end{array}$ & $\begin{array}{l}2006 / \\
2007\end{array}$ & 2008 \\
\hline \multicolumn{11}{|c|}{ Sowing dates (A) } \\
\hline $20^{\text {th }}$ October & 9.07 & 6.60 & 7.76 & 7.74 & 6.02 & 6.25 & 0.42 & 0.19 & 316.7 & 176.4 \\
\hline $10^{\text {th }}$ November & 8.99 & 6.40 & 7.65 & 7.69 & 5.80 & 5.91 & 0.26 & 0.23 & 538.8 & 249.0 \\
\hline $1^{\text {st }}$ December & 8.26 & 6.34 & 7.63 & 7.49 & 4.54 & 6.54 & 0.37 & 0.32 & 279.2 & 227.3 \\
\hline F-Test & NS & NS & NS & NS & NS & NS & ** & ** & ** & ** \\
\hline NLSD 0.05 & - & - & - & - & - & - & 0.01 & 0.01 & 2.97 & 7.12 \\
\hline \multicolumn{11}{|c|}{ Harvesting dates (B) } \\
\hline 135 days & 8.07 & 6.04 & 7.58 & 7.57 & 5.08 & 6.73 & 0.33 & 0.20 & 320.2 & 152.9 \\
\hline 150days & 8.44 & 7.50 & 7.83 & 7.82 & 6.08 & 6.13 & 0.33 & 0.25 & 395.5 & 222.2 \\
\hline 165 days & 9.81 & 5.80 & 7.63 & 7.53 & 6.83 & 6.85 & 0.39 & 0.29 & 419.0 & 277.5 \\
\hline F-Test & ** & ** & NS & NS & ** & ** & 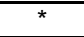 & * & ** & $\bar{*}$ \\
\hline NLSD 0.05 & 0.65 & 0.16 & - & - & 0.26 & 0.25 & 0.01 & 0.01 & 2.97 & 7.12 \\
\hline \multicolumn{11}{|c|}{ Varieties (C) } \\
\hline Sakh & .43 & 6.28 & 7.48 & 7.45 & 7.57 & 7.63 & 0.42 & 0.26 & 454.2 & 254.5 \\
\hline Sakha 3 & 36 & 5.98 & 7.99 & 7.86 & 4.19 & 4.85 & 0.22 & 0.23 & 272.3 & 178.6 \\
\hline Sakha 4 & 98 & 7.38 & 7.94 & 7.91 & 4.31 & 5.49 & 0.25 & 0.23 & 287.5 & 186.2 \\
\hline Giza 8 & 10.32 & 6.14 & 7.31 & 7.34 & 7.93 & 7.64 & 0.51 & 0.26 & 499.0 & 251.0 \\
\hline F-Test & ** & ** & * & * & * & ** & ** & * & ** & ** \\
\hline NLSD 0.05 & 0.70 & 0.46 & 0.42 & 0.39 & 0.31 & 0.43 & 0.01 & 0.01 & 3.39 & 5.15 \\
\hline \multicolumn{11}{|c|}{ Interaction F- Test } \\
\hline$A \times B$ & * & 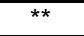 & NS & NS & * & * & ** & ** & 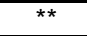 & ** \\
\hline$A X C$ & * & NS & NS & NS & * & * & ** & ** & ** & ** \\
\hline$B \times C$ & * & NS & NS & NS & 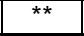 & * & 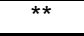 & ** & *夫 & ** \\
\hline$A \times B \times C$ & ** & * & NS & NS & NS & NS & NS & NS & ** & ** \\
\hline
\end{tabular}

These results are in agreement with those obtained by Sahsah et al. (1987), Shafshak et al. (1992), Mohamed et al. (1998), El-Deeb(1998), Amna et al. (2003) and El-Deeb and Abd El-Fatah (2006). 


\section{Performance of varieties.}

Data collected in Table 3 show means of number of capsules/ plant, number of seeds/ capsule, seed index , seed yield per plant and seed yield per faddan which were markedly in all the studied varieties. Over the two seasons, Giza 8 surpassed the other varieties significantly in number of capsules/ plant (10.32), seed index (7.93 and $7.64 \mathrm{gm})$, seed yield/ plant( 0.51 and $0.26 \mathrm{gm} /$ plant) and seed yield / faddan (499.0 gm / faddan) in the first season. Or both followed by Sakha 2, except number of capsules/ plant and number of seeds/ capsule. It could be concluded that sown Sakha 2 surpassed Sakha $357.2 \%$ and Sakha $4 \quad 49.6 \%$ as an average of both seasons. In addition, sowing Giza 8 variety surpassed Sakha 3 by $66.3 \%$ and Sakha 4 by $58.3 \%$ as an average of both seasons. However, the lowest average in seed index, seed yield per plant and seed yield per faddan was obtained sown Sakha 3 and Saka 4 in the two seasons. These results are in harmony with those obtained by Mohamed e. al. (1998), El-Shimy and Moawed (2000), Mostafa and Ashmawy (2003), El-Deeb and Abd El-Fatah (2006) and Abd El Daiem, (2009).

\section{Effect of interactions:}

The interaction between sowing dates and harvesting dates are presented in Table 4. It is clear that 1000 seed weight and seed yield per plant were significantly affected in both seasons The highest average seed index (7.12 and $7.23 \mathrm{gm})$ was obtained when sown on $20^{\text {th }}$ October and harvesting after 165 days from sowing date and seed yield per plant $(0.50$ and $0.40 \mathrm{gm} /$ plant) was obtained when sown on $20^{\text {th }}$ October and $1^{\text {st }}$ December and harvesting dates after 165 days from sowing respectively.

Concerning the interaction among sowing dates and studied flax varieties, the results presented in Table 5 clearly showed that seed index and seed yield per plant significantly affected by the interaction between sowing dates and studied varieties in both seasons. Highest seed index values was obtained from sowing Giza 8 or Sakha 2 varieties when sown on $20^{\text {th }}$ Oct. and $1^{\text {st }}$ Dec. sowing dates without significant differences between them in both seasons. The highest seed yield/ plant (0.66) was obtained from sowing Giza 8 variety on $20^{\text {th }}$ October in the first season and sown Giza 8 variety on $1^{\text {st }}$ December in the second season. However, the lowest seed yield/ plant was obtained from sowing Sakha 3 variety on $10^{\text {th }}$ November in the first season, and Sakha 4 variety on $20^{\text {th }}$ October in the second season. These finding are in good accordance with those obtained by Fontana et al. (1996), Mostafa (1999) and Saeidi and Khodambashi (2007).

Regarding to the interaction between harvesting dates and varieties on seed index, the results in Table 6 clearly indicated that seed index and seed yield per plant significantly affected due to the interaction among harvesting dates and flax varieties in both seasons. The highest seed index was produced from harvesting Giza 8 or Sakha 2 varieties after 150 or 165 days from sowing without significant differences between them in both seasons. In this respect, maximum seed yield per plant $(0.57$ and $0.33 \mathrm{gm} /$ plant $)$ was obtained from harvesting Giza 8 variety and harvesting it after 165 days from sowing in the first and second seasons, respectively. Whilst, the lowest seed index and seed yield/ plant was produced from harvesting Sakha 3 or Sakha 
Kandil, A. A. et al.

4 varieties after 150 or 135 days from sowing, respectively in both season. These finding are in good accordance with those obtained by El- Deeb and Abd-El- Fatah (2006).

Table 4: Means of 1000 seed weight and seed yield/ plant as affected by the interaction between sowing dates $x$ harvesting dates during 2006/2007 and 2007/2008 seasons.

\begin{tabular}{|c|c|c|c|c|c|c|c|c|c|c|c|c|}
\hline \multirow{3}{*}{\begin{tabular}{|l|} 
Characters \\
Treatments \\
$\begin{array}{l}\text { Harvesting } \\
\text { dates }\end{array}$ \\
\end{tabular}} & \multicolumn{6}{|c|}{1000 seed weight } & \multicolumn{6}{|c|}{ Seed yield/ plant } \\
\hline & \multicolumn{3}{|c|}{$2006 / 2007$} & \multicolumn{3}{|c|}{$2007 / 2008$} & \multicolumn{3}{|c|}{$2006 / 2007$} & \multicolumn{3}{|c|}{$2007 / 2008$} \\
\hline & $\begin{array}{l}20^{\text {th }} \\
\text { Oct }\end{array}$ & $\begin{array}{l}10^{\text {th }} \\
\text { Nov }\end{array}$ & $\begin{array}{c}1^{\text {st }} \\
\text { Dec }\end{array}$ & $\begin{array}{l}20^{\text {th }} \\
\text { Oct }\end{array}$ & $\begin{array}{l}10^{\text {th }} \\
\text { Nov }\end{array}$ & $\begin{array}{l}1^{\text {st }} \\
\text { Dec }\end{array}$ & $\begin{array}{l}20^{\text {th }} \\
\text { Oct }\end{array}$ & $\begin{array}{l}10^{\text {th }} \\
\text { Nov }\end{array}$ & $\begin{array}{l}1^{\text {st }} \\
\text { Dec }\end{array}$ & $\begin{array}{l}20^{\text {th }} \\
\text { Oct }\end{array}$ & $\begin{array}{l}10^{\text {th }} \\
\text { Nov }\end{array}$ & $1^{\text {st }}$ Dec \\
\hline After 135 days & 5.25 & 4.57 & 5.43 & 5.42 & 5.28 & 6.51 & 0.36 & 0.25 & 0.38 & 0.18 & 0.15 & 0.25 \\
\hline After 150 days & 5.70 & 6.53 & 6.01 & 6.12 & 5.89 & 6.37 & 0.40 & 0.23 & 0.36 & 0.18 & 0.27 & 0.31 \\
\hline After 165 days & 7.12 & 6.30 & 7.08 & 7.23 & 6.57 & 6.75 & 0.50 & 0.30 & 0.36 & 0.21 & 0.27 & 0.40 \\
\hline$F$ test & & * & & & * & & & * & & & * & \\
\hline NLSD 5\% & & 0.60 & & & 0.61 & & & 0.01 & & & 0.01 & \\
\hline
\end{tabular}

Table 5: Means of 1000 seed weight and seed yield/ plant as affected by the interaction between sowing dates $\times$ varieties during 2006/ 2007 and 2007/ 2008 seasons.

\begin{tabular}{|c|c|c|c|c|c|c|c|c|c|c|c|c|}
\hline \multirow{3}{*}{\begin{tabular}{|l|} 
Characters \\
Treatments \\
Varieties
\end{tabular}} & \multicolumn{6}{|c|}{1000 seed weight } & \multicolumn{6}{|c|}{ Seed yield/ plant } \\
\hline & \multicolumn{3}{|c|}{$2006 / 2007$} & \multicolumn{3}{|c|}{$2007 / 2008$} & \multicolumn{3}{|c|}{$2006 / 2007$} & \multicolumn{3}{|c|}{$2007 / 2008$} \\
\hline & $\begin{array}{l}20^{\text {th }} \\
\text { Oct }\end{array}$ & $\begin{array}{l}10^{\text {th }} \\
\text { Nov }\end{array}$ & $1^{\text {st }} \mathrm{De}$ & $\begin{array}{l}20^{\text {th }} \\
\text { Oct }\end{array}$ & $\begin{array}{l}10^{\text {th }} \\
\text { Nov }\end{array}$ & $1^{\text {st }} \mathrm{De}$ & $\begin{array}{l}20^{\text {th }} \\
\text { Oct }\end{array}$ & $\begin{array}{l}10^{\text {th }} \\
\text { Nov }\end{array}$ & $1^{\text {st }} \mathrm{De}$ & $\begin{array}{l}20^{\text {th }} \\
\text { Oct }\end{array}$ & $\begin{array}{l}10^{\text {th }} \\
\text { Nov }\end{array}$ & $1^{\text {st }} \mathrm{De}$ \\
\hline Sakha 2 & 8.02 & 7.57 & 7.12 & 7.64 & 7.45 & 7.81 & 0.50 & 0.31 & 0.45 & 0.23 & 0.21 & 0.34 \\
\hline Sakha 3 & 3.99 & 4.25 & 4.31 & 5.00 & 4.74 & 4.80 & 0.27 & 0.14 & 0.25 & 0.17 & 0.22 & 0.30 \\
\hline Sakha 4 & 3.91 & 4.13 & 4.88 & 4.14 & 4.31 & 5.75 & 0.24 & 0.20 & 0.30 & 0.14 & 0.28 & 0.27 \\
\hline Giza 8 & 8.17 & 7.24 & 8.39 & 7.97 & 7.16 & 7.82 & 0.66 & 0.38 & 0.47 & 0.21 & 0.21 & 0.37 \\
\hline F test & & * & & & * & & & ** & & & 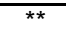 & \\
\hline NLSD 5\% & & 0.7 & & & 0.75 & & & 0.01 & & & 0.01 & \\
\hline
\end{tabular}

Table 6: Means of 1000 seed weight and seed yield/ plant as affected by the interaction between harvesting dates $\times$ varieties during 2006/2007 and 2007/2008 seasons.

\begin{tabular}{|c|c|c|c|c|c|c|c|c|c|c|c|c|}
\hline \multirow[t]{2}{*}{ Characte } & \multicolumn{6}{|c|}{1000 seed weight } & \multicolumn{6}{|c|}{ Seed yield/ plant } \\
\hline & \multicolumn{6}{|c|}{ Harvesting dates } & \multicolumn{6}{|c|}{ Harvesting dates } \\
\hline \multirow{2}{*}{\begin{tabular}{|l|} 
Treatme \\
Varieties
\end{tabular}} & \multicolumn{3}{|c|}{$2006 / 2007$} & \multicolumn{3}{|c|}{$2007 / 2008$} & \multicolumn{3}{|c|}{$2006 / 2007$} & \multicolumn{3}{|c|}{$2007 / 2008$} \\
\hline & $\begin{array}{l}135 \\
\text { days }\end{array}$ & $\begin{array}{c}150 \\
\text { days }\end{array}$ & $\begin{array}{c}165 \\
\text { days }\end{array}$ & $\begin{array}{c}135 \\
\text { days }\end{array}$ & $\begin{array}{c}150 \\
\text { days }\end{array}$ & $\begin{array}{r}165 \\
\text { days }\end{array}$ & $\begin{array}{c}135 \\
\text { days }\end{array}$ & $\begin{array}{c}150 \\
\text { days }\end{array}$ & $\begin{array}{r}165 \\
\text { days }\end{array}$ & $\begin{array}{c}135 \\
\text { days }\end{array}$ & $\begin{array}{r}150 \\
\text { days }\end{array}$ & $\begin{array}{r}165 \\
\text { days }\end{array}$ \\
\hline Sakha 2 & 6.32 & 7.50 & 8.91 & 6.63 & 7.83 & 8.44 & 0.41 & 0.39 & 0.46 & 0.21 & 0.29 & 0.28 \\
\hline Sakha 3 & 3.55 & 4.61 & 4.39 & 4.44 & 4.62 & 5.47 & 0.22 & 0.20 & 0.24 & 0.19 & 0.23 & 0.27 \\
\hline Sakha 4 & 3.89 & 4.20 & 4.83 & 4.34 & 4.39 & 4.74 & 0.22 & 0.25 & 0.28 & 0.17 & 0.25 & 0.27 \\
\hline Giza 8 & 6.57 & 8.02 & 9.20 & 6.53 & 7.67 & 8.75 & 0.47 & 0.47 & 0.57 & 0.21 & 0.24 & 0.33 \\
\hline F test & \multicolumn{3}{|c|}{ ** } & \multicolumn{3}{|c|}{ * } & \multicolumn{3}{|c|}{ ** } & \multicolumn{3}{|c|}{ ** } \\
\hline NLSD 5\% & \multicolumn{3}{|c|}{0.77} & \multicolumn{3}{|c|}{0.75} & \multicolumn{3}{|c|}{0.01} & \multicolumn{3}{|c|}{0.02} \\
\hline
\end{tabular}


Table 7: Means of number of capsules per plant and seed yield/faddan as affected by the interaction among sowing dates, harvesting dates and varieties during both seasons.

\begin{tabular}{|c|c|c|c|c|c|c|c|c|c|c|c|c|c|}
\hline \multirow{2}{*}{\multicolumn{2}{|c|}{\begin{tabular}{|l|} 
Characters \\
Treatments
\end{tabular}}} & \multicolumn{6}{|c|}{ number of capsules per plant } & \multicolumn{6}{|c|}{ Seed yield/ faddan } \\
\hline & & \multicolumn{3}{|c|}{$\begin{array}{c}\text { Sowing dates } \\
2006 / 2007\end{array}$} & \multicolumn{3}{|c|}{$\begin{array}{c}\text { Sowing dates } \\
2006 / 2007\end{array}$} & \multicolumn{3}{|c|}{$\begin{array}{c}\text { Sowing dates } \\
2006 / 2007\end{array}$} & \multicolumn{3}{|c|}{$\begin{array}{c}\text { Sowing dates } \\
2007 / 2008 \\
\end{array}$} \\
\hline 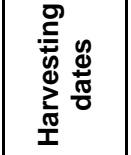 & 离 & $\begin{array}{l}\overline{0} \\
\text { o } \\
\overline{\text { స్ }}\end{array}$ & $\begin{array}{l}\text { o } \\
\text { ż } \\
\text { 晋 }\end{array}$ & 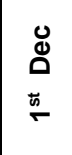 & $\begin{array}{l}\overline{0} \\
0 \\
\overline{0}\end{array}$ & $\begin{array}{l}\text { o } \\
\text { ż } \\
\text { 严 }\end{array}$ & 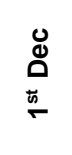 & 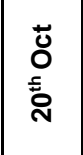 & $\begin{array}{l}\text { o } \\
\mathbf{z} \\
\text { 홍 }\end{array}$ & $\begin{array}{l}0 \\
\text { Dू } \\
\text { ñ } \\
\text { - }\end{array}$ & $\begin{array}{l}\overline{0} \\
\text { o } \\
\bar{\Xi} \\
\text { స్ }\end{array}$ & $\begin{array}{l}\text { 으 } \\
\text { 产 } \\
\text { 은 }\end{array}$ & $\begin{array}{l}\text { Dू } \\
\text { Q } \\
\vec{\omega}\end{array}$ \\
\hline \multirow{4}{*}{$\begin{array}{l}\text { After } 135 \\
\text { days }\end{array}$} & tha 2 & 11 & 6 & 8.30 & 71 & 56 & 6.58 & 263.7 & 460.3 & 97.3 & 09.6 & 132.6 & 57 \\
\hline & Sakha & 0 & 0 & 7.83 & 5.7 & 6.17 & 3.72 & 186.2 & 345 & & & 1 & 149 \\
\hline & Sakha 4 & 7.78 & 6.53 & 6.70 & 7.86 & 7.56 & 5.58 & 164.2 & 387.3 & 2 & 10 & 178.9 & 155. \\
\hline & Giza 8 & 8.38 & 15.10 & 10.77 & 5.80 & 6.26 & 4.96 & 298.4 & 593.6 & 456.6 & 74.8 & 154.2 & 291. \\
\hline \multirow{4}{*}{$\begin{array}{l}\text { After 150 } \\
\text { days }\end{array}$} & Sakha 2 & 7.76 & 7.06 & 7.31 & 7.13 & 9.00 & 7.10 & 398.5 & 802.9 & .4 & 175.5 & 302.9 & 354. \\
\hline & Sakha 3 & 9.13 & 7.63 & 8.03 & 8.16 & 6.80 & 7.63 & 240.3 & 410.9 & 138.1 & 144.3 & 203.7 & 156.1 \\
\hline & Sakha 4 & 6.23 & 10.50 & 10.86 & 10.03 & 8.12 & 5.83 & 240.1 & 423.9 & 199.5 & 130.3 & 240.7 & 174.6 \\
\hline & Giza 8 & 10.46 & 8.80 & 7.50 & 7.26 & 7.30 & 5.60 & 389.8 & 713.7 & 469.4 & 153.7 & 317.6 & 174.4 \\
\hline \multirow{4}{*}{$\begin{array}{l}\text { After } 165 \\
\text { days }\end{array}$} & Sakha 2 & 13.66 & 6.02 & 5.93 & 5.13 & 3.40 & 5.96 & 427.4 & 725.6 & 392.9 & 343.9 & 338.5 & 274.9 \\
\hline & Sakha 3 & 11.36 & 9.66 & 6.98 & 4.48 & 5.67 & 5.47 & 381.7 & 450.0 & 119.3 & 245.5 & 324.3 & 156.7 \\
\hline & Sakha 4 & 12.00 & 10.16 & 10.06 & 6.56 & 5.56 & 9.30 & 361.9 & 435.8 & 164.5 & 245.9 & 310.5 & 135.2 \\
\hline & Giza 8 & 9.43 & 13.65 & 8.83 & 5.33 & 4.40 & 8.33 & 448.8 & 716.1 & 404.3 & 286.5 & 359.8 & 308. \\
\hline \multicolumn{2}{|l|}{ F Test } & \multicolumn{3}{|c|}{ ** } & & \multirow{2}{*}{\multicolumn{3}{|c|}{$\begin{array}{c}* * \\
10.1\end{array}$}} & \multirow{2}{*}{\multicolumn{3}{|c|}{1}} \\
\hline \multicolumn{2}{|l|}{ NLSD 0.05} & \multicolumn{3}{|c|}{2.89} & \multicolumn{2}{|r|}{1.83} & & & & & \multicolumn{2}{|r|}{17.1} & \\
\hline
\end{tabular}

The interaction between sowing and harvesting dates of the studied flax varieties significantly affected number of capsules/ plant and seed yield/ faddan as presented in Table 7. The highest seed index were obtained by sowing flax Giza 8 or Sakha 4 varieties on $10^{\text {th }}$ November and $20^{\text {th }}$ October and harvesting after 135 or 150 days from sowing, respectively without significantly differences between them in the first season. Heights seed yield/ faddan was obtained from sowing Giza 8 variety on $10^{\text {th }}$ November and harvesting after 165 days from sowing in the first season. (716.1 gm) However, the lowest number of capsules/ plant was obtained from sowing Sakha 2 variety on $20^{\text {th }}$ October and harvesting dates after 135 days from sowing dates and the lowest seed yield/faddan was obtained from sowing Sakha 3 variety on $1^{\text {st }}$ December and harvesting after 165 days from sowing in the first season and from sown the flax Giza 8 variety on $20^{\text {th }}$ October and harvesting after 135 days in the second season. These results are in harmony with those obtained by Mohamed et al. (1998) Jankauskien and Mikelionis (2001) and El-Deeb and Abd El-Fatah (2006).

\section{REFERENCES}

Abd El Daiem, M.A. (2009). Resonse of some flax genotypes to sowing dates and micro elements fertilization. Ph.D. Thesis, Fac. Agric., Al-Azhar Univ

Abd-El-Daiyme, M.A. ; E.A. Maowed and M.M.M. Hussein (2007). Response of Sakha2 flax variety to farmyard manure and plant density under different harvesting dates. J. Agric. Sci., Mansoura Univ., 32(3):1601-1610. 
Abdel-Fatah, A.A.E (1994). Agricultural studied on flax crop .M.Sc. Thesis, Fac., Agric., Kafr El-Shelik, Tanta Univ., Egypt.

Abou-Zaied, T.A. and A.M. Mousa (2007). Effect of different NPK treatmes on yield and yield components of two flax varieties. J. Agric. Sci. Mansoura. Univ., 32 (10): 8057-8064.

Amna, H.H.E ; M.A. Tag el Din and H.A.M. Sharaf El- Deen (2003). Effect of some flax genotypes and harvesting dates on seed chemical composition, yield and fiber quality. Moshtohor Annals of Agric. Sci., 41(1): 19-37

Dixit, T.P. ; S.K. Chourasia ; P.V.A pillai and R.A. Khan (1994). Assessment of flax flexibility of sowing time of linseed (linum usitatissimum L.) varieties under double cropping system in Tawa commend. Indian J. of Agron., 39(1):105-109.

El-Deeb, E.A. and A.A. Abd- El Fatah (2006). Effect of sowing and harvesting dates on yield and its quality for some flax varieties. J. Agric. Sci., Mansoura Univ., 31(9): 555- 566.

El-Deeb,E.A.(1998). Response of some flax varieties to phosphatic fertilizer and harvesting dates .M. Sc. Thesis ,Fac .of Agric .,Moshtohor ,Zagazig Univ.

El-Kady, E.A.F ; S. E. Shafshak ; F. I. Gab-Allah and M. E. A. Kineber (1995). Effect of seeding rates on yield and its components of six promising flax genotypes under saline conditions. J. Agric. Sci. Mansoura Univ., 20(2): 593-602.

El-Shimy, G.H. and E. A. Moawed (2000). Effect of different potassium and nitrogen fertilizer levels on Giza 8 and Viking flax varieties. J. Agric. Sci., Mansoura Univ., 25 (10): 5993- 6007.

Fontana, F. ; D. Cremaschi ; C. Vender ; C. Maestrini and L. Natarelli (1996). Comparison of two spring sowing dates for linseed (linum usitatissimum L.) cultivars. Rivista di Agronomia. 30 (3): 248-251.

Gomaz, K.A. and A.A. Gomaz (1984). Statitstical procedures for agricultural researches. John Wiley and Sons. Inc. New York, $2^{\text {nd }}$ Ed.,68 p.

Jankauskiene, Z. and S. Mikelionis (2001). The influence of fibre flax varieties' ripeness and harvesting method on fiber output and quality. Zemdirbyste, Mokslo Darbai. 2001; 76: 47-54.

Kineber, M.E.A. (1994). Evaluation of some new promising flax varieties strains under soil conditions. Ph.D. Thesis, Fac. Agric. Moshtohor, Zagazig Univ., Egypt.

Mohamed, A.A.E ; M.E.A. Kinber and E.A.F.El-Kady (1998). Effect of sowing and harvesting date on yield of flax genotypes .J.Adv. Agric.Res., AlexSaba-Basha, 3 (1): 31-42

Moawed, E.A. (1996). Agronomic studies on flax cultivars. Ph. D. Thesis, Fac. Agric., Zagazig Univ., Egypt.

Mostafa, S.H.A (1990). Response of flax to seeding rate and harvesting time .M. Sc. Thesis, Fac. Agric., Mosthohor, Zagazig Univ. (Banha Branch).

Mostafa, A.S. (1999). Comparative studies between some new flax lines and commercial variety Giza 7 M..Sc. Thesis Fac, Agric, Mansoura Univ. 
Mostafa, S.H.A and F. Ashmawy (2003). Comparative study for traits of some flax genotypes using factor and cluster analyses Moshtohor, Egypt. Faculty of Agriculture, Zagazig Univ. 41(1): 1-18.

Saeidi, G. and M. Khodambashi (2007). Evaluation of agronomic traits of edible oil genotypes of flax at two seeding dates in Shahrekord. $J$ of Sci. and Technology of Agric. and Natural Res.,10(4) (B): 309-321.

Salama, A.M.(1996). Influence of planting date and nitrogen fertilizer levels on agronomic characteristics of three flax cultivars. J. Agric. Sci. Mansoura Univ., 21 (3): 881- 891.

Sahsah ,A.I ; A. Hella and S.M. Gaafar (1987). Effect of harvesting dates on growth, yield and its quality of flax(linum usiatatissimum L.) .Ann Agric. Sci., Moshtohor,25 (3):1171-1181.

Shafshak, S.E. ; T.N. El-Din ; A.M. Hella ; S.H.A. Mostafa (1992). Effect of seeding rates and harvesting times on flax yield. Egypt. J. of Agric. Res., 70(4): 1205-1215

Waller, R.A. and D.B. Dunckan (1969). Abays rule for the symmetric multiple comparison problem. J. Amer. Assoc., 64: 1484-1503.

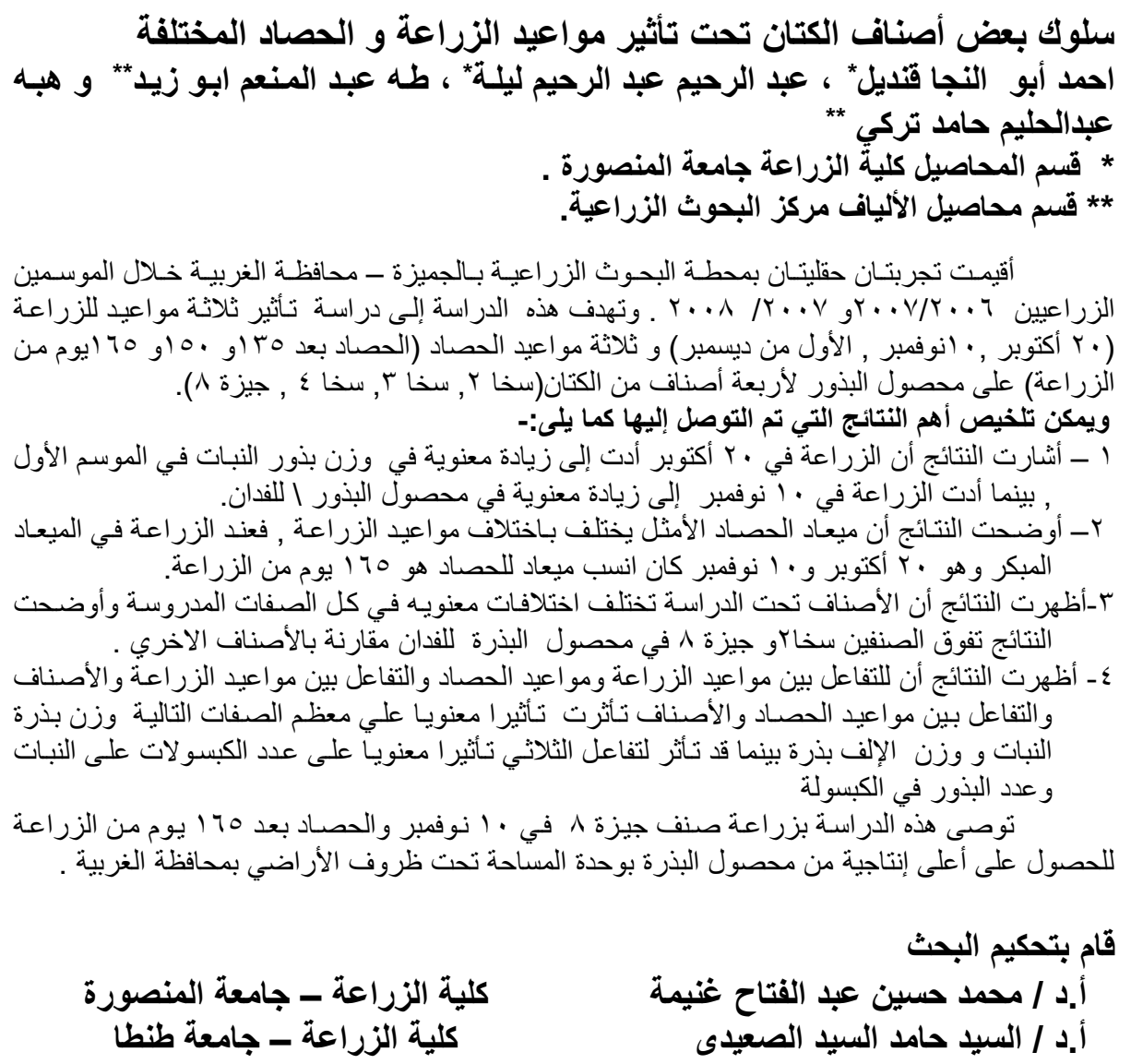

كلية الزراعة - جامعة المنصورة كلية الزراعة - جامعة طنطا جلماكة 